\title{
Arg304Gln (FVII Padua) coagulation disorder in a patient with Down Syndrome (trisomy 21): a remarkable observation from Argentina
}

\author{
Antonio Girolami ${ }^{1}$, Maria Silvia Farhat ${ }^{2}$, Cecilia Hayward ${ }^{3}$, Silvia Ferrari ${ }^{1}$ and Eleonora B Rossi $^{3}$ \\ ${ }^{1}$ University of Padua Medical School, Department of Medicine, Padua, Italy \\ ${ }^{2}$ Hospital Angel Cruz Padilla, Seccion de Hemostasia, Tucuman, Argentina \\ ${ }^{3}$ Laboratorio de Salud Publica, Seccion de Hemostasia, Tucuman, Argentina
}

\begin{abstract}
Objectives: to investigate the peculiar association between Down Syndrome (Trisomy 21) and a FVII defect (FVII Padua) in a family from Argentina.

Patients and Methods: The propositus is a 45 year old man who manifested the typical features of Down Syndrome and an asymptomatic FVII defect.

Results: FVII level was low using rabbit brain thromboplastin but it was near normal using recombinant reagents and perfectly normal with OX brain thromboplastin.

Molecular analysis revealed that the patient was homozygote for the Arg304Gln mutation in exon 8 (FVII Padua), whereas his parents were heterozygotes for the same mutation. A younger brother of the propositus was normal.

No bleeding or thrombosis was present in the propositus and in his family members.

Conclusions: The patient is the first patient with Down Syndrome found to be associated with a rare clotting defect such as FVII deficiency. So far only three associations with Hemophilia A or B had been investigated. The significance of the peculiar association is discussed.
\end{abstract}

\section{Introduction}

FVII deficiency is the most frequent defect encountered among the rare coagulation disorders. A prevalence of about 1:500.000 thousand people has been estimated and the defect has been described in several parts of the world [1].

FVII Padua (Arg304Gln was first described in 1978 in Italy and subsequently reported in other countries [2,3]. It is a FVII variant characterized by the presence of a variable FVII activity according with the tissue thromboplastin used in the assay system. The FVII activity level is low if a rabbit brain thromboplastin is used whereas it is near normal if human recombinant thromboplastins are used and it is perfectly normal if the OX brain thromboplastin is employed in the assay system [2]. FVII antigen is perfectly normal [2].

Bleeding manifestations are limited or even absent. On the contrary, venous thrombosis has been reported in these patients [4].

The defect has been described in association with other morbid conditions but never with Down Syndrome. Trisomy 21 or Down Syndrome is the most frequent genetic defect with a prevalence of about 1:1000 live births and it has been encountered all over the world $[5,6]$.

The opportunity we have to study a patient with Down Syndrome and a prolonged PT that was not corrected by the administration of Vit. K prompted further studies. These led to the identification in the propositus of a congenital FVII defect (FVII Padua).

The association between Down Syndrome and a congenital coagulation disorders is an extremely rare event. So far only three patients with Down syndrome and hemophilia A or Hemophilia B have been reported [7-9]. The association with other coagulation factor has never been reported.

The purpose of the present paper is the one to report the study of the unique extraordinary association between Down Syndrome and FVII Padua.

\section{Family study and methods}

The propositus is a 46 year old male who lives in northewestern Argentina (Figure 1).

Parents were not consanguineous and had, apparently, a spanishportuguese background. The mother was 30 year old when the propositus was born. The diagnosis of Down Syndrome due to a Trisomy 21 was established during the first year of life in Argentina. Despite the diagnosis, the child had no major problem during childhood. The typical physical features of Down syndrome were: a moderate mental impairment together with the usual facial expression, speech impairment, and a limited growth. However, he was always affectionally and socially involved with family members and friends.

*Correspondence to: Antonio Girolami, Dept of Medicine, Via Ospedale, 105, Padua-35128, Italy, Tel 00390498213026; Fax 0039049657391; E-mail: antonio. girolami@unipd.it

Key words: Down Syndrome, Trisomy 21, FVII, FVII Padua, Arg304Gln

Received: April 14, 2020; Accepted: April 24, 2020; Published: April 27, 2020 


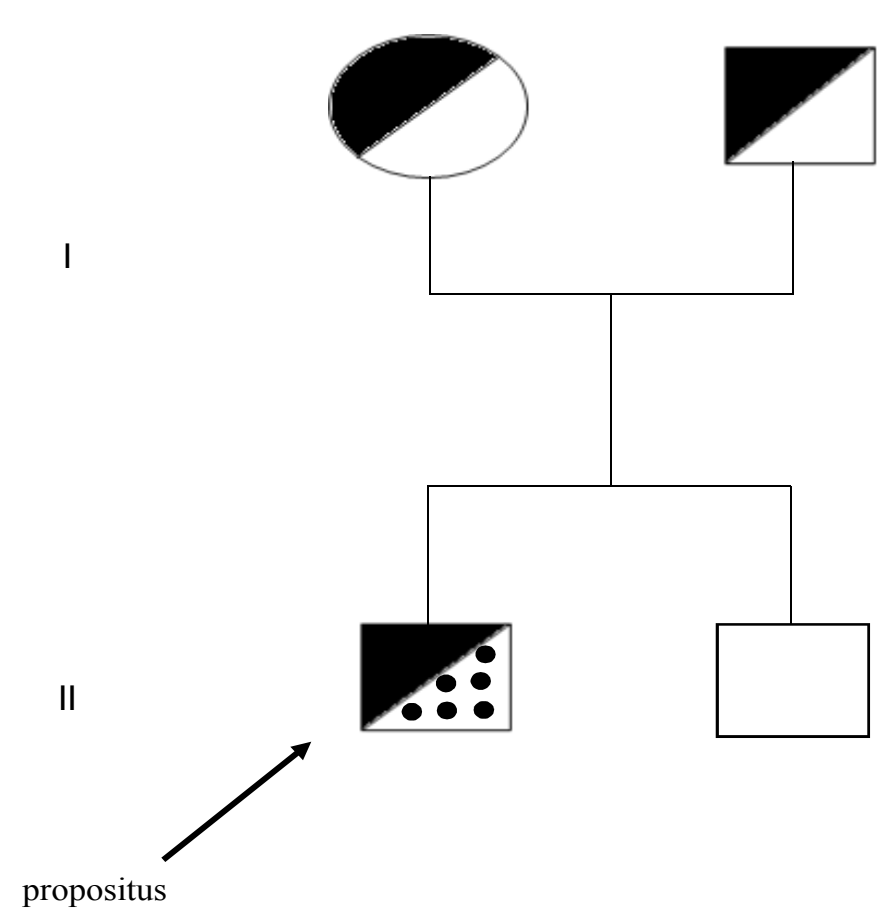

Figure 1. Family pedigree. The propositus, affected by Down Syndrome and homozygosity for the Arg304Gln mutation (FVII Padua) is indicated by an arrow. The parents are both heterozygotes for the Arg304Gln mutation. The younger brother is normal.

The patient never presented undue bleeding. Tooth extractions were carried out without any complication. At the age of 19 he presented hematuria without any apparent cause. At that time a PT 20\% of normal was found. The defect did not respond to Vit K administration. Since the hematuria subsided spontaneously, no further studies were carried out and a tentative diagnosis of liver damage was formulated.

The patient felt well for several years despite the limitation due to the basic abnormality. He was involved in several manual activities that he accomplished with success and satisfaction. At the age of 41, a laboratory control revealed a PT of $15 \%$ of normal and a FVII level of $2 \%$, whereas FII, FV, FVIII, FIX and FX were normal. Another FVII assay, in another laboratory, was $13 \%$. A diagnosis of FVII deficiency was then formulated.

At the age of 45 the patient presented with episodes of dyspepsia and vomit. Multiple gall bladder stones were discovered and surgery was indicated. A preparatory evaluation of the clotting system revealed a PT of $18 \%$ and a FVII of $1 \%$ of normal.

Tests were then repeated in another hospital using a human recombinant thromboplastin and a PT of $89 \%$ of normal together with a FVII level of $49 \%$ were obtained.

Surgery was then carried out without the protection of Prothrombin Complex concentrates which had been prepared in case of excessive bleeding. No complication occurred. The patient has never had a venous or arterial thrombotic event. On the suspicion of a FVII abnormality samples were sent to Padua for further studies.

Coagulation tests were carried out in Tucuman and in Padua as previously reported [2]. aPTT and PT were carried out using standard procedures. The reagents used for the aPTT was supplied by Instrumentation Laboratory, Milan, Italy. Five reagents were used for the PT and the FVII assays, namely: two rabbit brain thromboplastins (Thromboplastin HS, Instrument Lab, Milan and Neoplastin plus, Stago
Laboratories, Asniers, France); a reagent obtained from human placenta (Thromborel S, Dade-Behring, Marburg, Germany); one human recombinant thromboplastin (Recombiplastin 2G, Instrumentation Laboratory, Milan, Italy) and an ox brain thromboplastin (Thrombotest, Nygard Laboratories, Norway).

Factor VII clotting assays were carried out on 1:10 diluited plasma using known FVII deficient plasma as substrate and the different thromboplastins. FVII antigen level was studied by an Elisa method (Asserachrom FVII, Stago Laboratories, Asniers, France). Molecular studies were carried out as previously reported [10], DNA was extracted from dried thick drops of whole blood blotted on Whatman paper. For this purpose we used the kit (QiAmp DNA minikit) supplied by QIAGEN Laboratories (Qiagen s.r.l., Milan, Italy). Amplification of exons 1 to 8 and respective spice junctions of the FVII gene were performed using oligonucleotide primers kindly supplied by Dr. James H. (Tyler, Tx, U.S.A.) or acquired from Invitrogen (Carlsbad, Ca, U.S.A.).

Mutational analysis was performed by polymerase chain reaction (PCR) amplification using oligo 8AF (5'-GAGGTGGCAGGTGGTGGAAA- 3'), 8AR (5'CGGCACAGAACATGTACTCC-3') 8BF (5'- TGATGACCCAGGACTGCCT-3'), 8BR (5'- GGGATTTGGTGCCAGGACA- 3'). PCR was carried out in a total volume of $15 \mu \mathrm{L}$ with $50 \mathrm{ng}$ of genomic DNA, $10 \mathrm{mM}$ of each primer, and $9 \mu \mathrm{l}$ of PCR Master Mix, 2X (Promega, Madison, Wisconsin, U.S.A.). After an initial denaturation step at $95^{\circ} \mathrm{C}$ for 5 minutes, amplification was performed for 35 cycles (denaturation at $95^{\circ} \mathrm{C}$ for 1 minute, annealing at $57^{\circ} \mathrm{C}$ for 1 minute, and extension at $72^{\circ} \mathrm{C}$ for 2 minutes). PCR products were bidirectionally sequenced using the ABI PRISM BigDye Terminator Cycle Sequencing Ready Reaction Kit and ABI3130 Genetic Analyzer (Applied Biosystems, Foster City, Ca, U.S.A.).

\section{Results}

Main results of the coagulation study are summarized in Table 1. Platelets and bleeding time are normal. PTT wass also normal., on the contrary, PT was variably prolonged using rabbit brain thromboplastins but was near normal or normal using a recombinant thromboplastin, or an ox-brain preparation. The prolonged PT obtained with the rabbit brain thromboplastin was corrected by the addition of normal plasma. Similarly, FVII assay was also low with the rabbit brain thromboplastin but it was near normal (49\%) with the recombinant preparation and completely normal (100\% of normal) using the ox-brain thromboplastin.

All other coagulation factors were within the normal limits. The parents had a slightly prolonged or a borderline PT and FVII levels around $50-60 \%$ of normal using rabbit brain thromboplastins. They had near normal or normal FVII levels using the recombinant preparations.

Molecular biology studies revealed that the propositus resulted to be homozygote for Arg304Gln mutation in exon 8. The parents were heterozygotes for the same mutation and had no bleeding tendency. The brother was normal (Figure 2). Furthermore, no family member had thrombotic events.

\section{Discussion}

Trisomy of chromosome 21 (Down Syndrome) is supposed to have a prevalence of about 1 in 1000 live births [5]. The abnormality is frequent in Argentina [6]. This family had the peculiarity that the mother of the propositus was 29 years old at the time of conception and that a younger brother of the propositus was normal. 
Table 1. Coagulation studies carried out in the propositus

\begin{tabular}{|c|c|c|}
\hline Test & Result & Normal values \\
\hline Platelet count & $190 \times 1000$ & $150-300 \times 1000$ \\
\hline Bleeding time & $<5 \mathrm{~min}$ & $<5$ minutes \\
\hline PTT & $37 \mathrm{sec}$ & $32-40 \mathrm{sec}$ \\
\hline PT & $54 \mathrm{sec}(14 \%)$ & $14-15 \mathrm{sec}$ \\
\hline TT & $16 \mathrm{sec}$ & $15-20 \mathrm{sec}$ \\
\hline FVII activity & $1 \%$ & $85-115 \%$ \\
\hline FVII activity & $1 \%$ & $85-115 \%$ \\
\hline FVII activity & $41 \%$ & $85-115 \%$ \\
\hline FVII activity & $49 \%$ & $85-115 \%$ \\
\hline FVII activity & $100 \%$ & $85-115 \%$ \\
\hline FVII antigen & $102 \%$ & $85-125 \%$ \\
\hline RII, FV,FVIII, FIX, FX, FXI, FXII & normal & $85-115 \%$ \\
\hline
\end{tabular}

1. Propositus

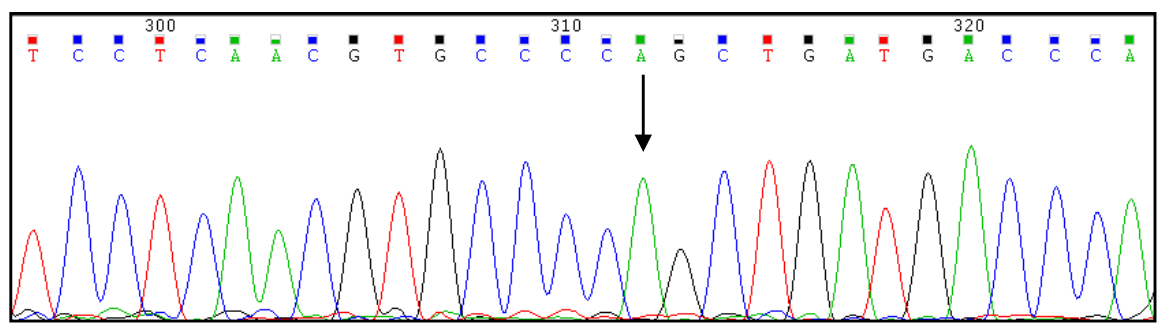

\section{Mother}

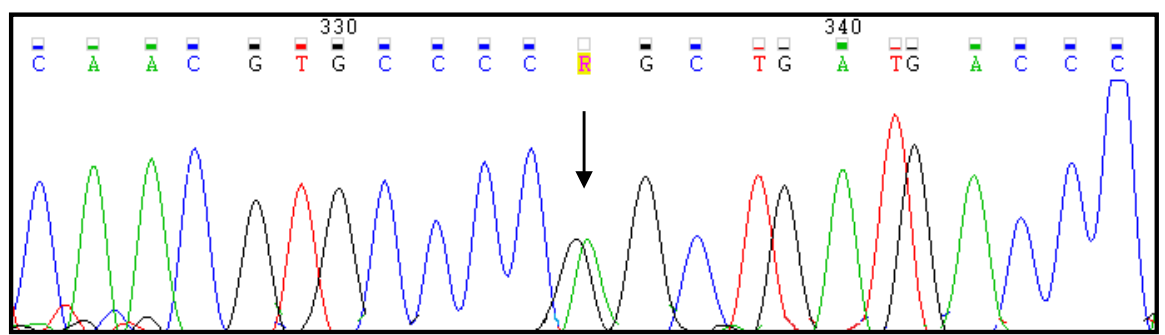

\section{Father}

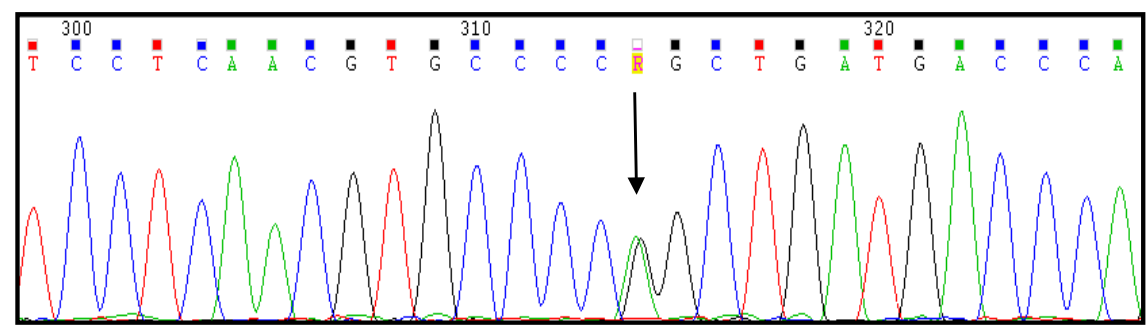

\section{Brother}

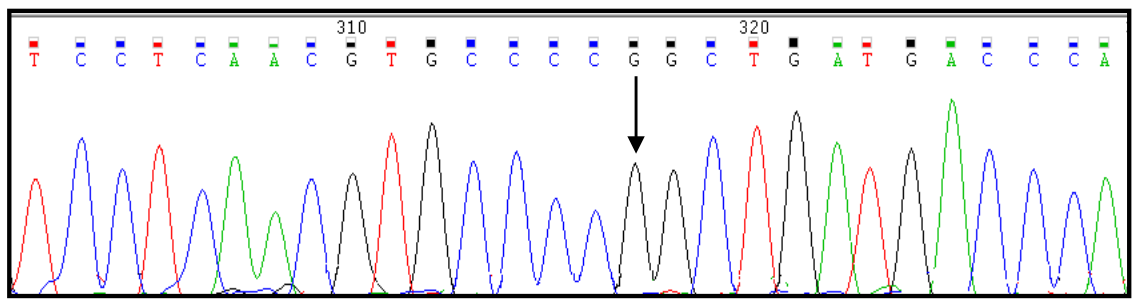

Figure 2. DNA sequencing pattern in the propositus who is homozygote for the Arg304Gln mutation (top); his mother and his father, who are heterozygotes, (middle tracings) and his normal brother (bottom). Arrows indicate site of mutation in exon 8. 
Due to this relatively high incidence, Down Syndrome has been associated with several conditions.

However, the association with congenital bleeding disorders seems extremely rare. Only two patients with severe Hemophilia A $[7,8]$, and one patient with Hemophilia B have been described [9]. In a general paper on the hematological conditions found in patients with Down Syndrome bleeding disorders are not even mentioned [10].

Down Syndrome has been found to be occasionally associated also with prothrombotic conditions. A 1 year old girl was found to be heterozygote for FV Leiden and homozygote for methylentetrahydrofolato reductase (MTHFR) deficiency and a 19 year old woman, heterozygote for FV Leiden $[11,12]$. In this regard it has to be underlined that for the majority of patients no information is available [13-15].

The present association with a rare coagulation disorder such a FVII defect is unique. Despite the two abnormalities the patient is still in fair condition. This is the third proven case of FVII Padua found in Argentina [16,17]. Similar cases may exist but there is no proof.

FVII Padua has been reported to be associated with venous thrombosis but the patient never complained of such condition [4]. This is of relevance since Down syndrome has been also frequently associated with thrombotic events, mainly venous [18-20].

The rarity of the association between Down Syndrome and clotting disorders is surprising. If one takes into account that Down syndrome (chromosome 21) is present in about 1 in 1000 live births and that the two hemophilias (chromosome X) in about 1:5000 people, one would expect a more frequent clinical association.

The only 3 cases presented in the literature are probably less than what could be expected. Since patients with hemophilia A or B do bleed, it is unlikely that such a combination of defects could go undetected. The association with other clotting defects, particularly if at the heterozygote level, may go undetected for the frequent lack of bleeding symptoms. The PT prolongation may be wrongly attributed to liver damage and/or to poor Vit $\mathrm{K}$ intake when it could have been due to the association with a mild deficiency of FII, FV, FVII or FX. Since FVII deficiency has a prevalence of 1:500.000 whereas that of the other rare coagulation disorders, namely FII, FV, FX,FXIII is about $1: 1.500 .000$, it is not surprising that the first association of DS occurred with the former. Association with the latter defects may never occur.

No observation has been demonstrated that chromosome 21 is involved with the chromosomes responsible for clotting factors defects.

Interestingly, Zergollern, et al. [7], reported that the patient with hemophilia A and Down syndrome had lower levels of FVIII (less than $1 \%)$ as compared with a brother who had only hemophilia A but with a FVIII level of $2.8 \%$ of normal. The significance of this observation remained unexplained and in contrast with the observation that hemophilia severity is uniform in the families affected. If confirmed it could indicate that the presence of Down syndrome could aggravate the clotting defect.

The presence of Trisomy 21 does not seem to influence the FVII clotting defect. In fact, the typical clinical and laboratory picture of FVII Padua defect was present in the propositus.

The present study indicates that Down Syndrome may be associated also with chromosome 13 that controls FVII.

Finally, it is worth noting that the patient never presented with a thrombotic event, despite the presence of two conditions, namely FVII
Padua and Down Syndrome which are variably, but surely associated with thrombosis, especially venous thrombosis [4,21-23].

\section{Compliance with ethical standards}

1) The study was carried out according to the Helsinki convention. The propositus and his parents were duly informed about the investigation and gave their consent.

2) The authors declare that there are no conflict of interest.

\section{Acknowledgement}

This study was supported in part by the "Associazione Emofilia ed altre coagulopatie delle Tre Venezie".

\section{References}

1. Perry DJ (2002) Factor VII Deficiency. Br J Haematol 118: 689-700. [Crossref]

2. Girolami A, Fabris F, Dal Bo Zanon R, Ghiotto G, Burul A (1978) Factor VII Padua: a congenital coagulation disorder due to an abnormal factor VII with a peculiar activation pattern. J Lab Clin Med 91: 387-395.

3. Girolami A, Berti de Marinis G, Bonamigo E, Allemand E (2011) Worldwide diffusion of FVII Arg304Gln coagulation defect (FVII Padua). Eur J Haematol 86: 135-139.

4. Girolami A, Cosi E, Ferrari S, Girolami B, Randi ML (2019) Thrombotic Events in Homozygotes with a Proven or Highly Probable Arg304Gln Factor VII Mutation (FVII Padua): Only Limited Replacement Therapy is Needed in Case of Surgery. Cardiovasc Hematol Disord Drug Targets 19: 233-238.

5. Nazer HJ, Cifuentes L, Estudio epidemiológico global del síndrome de Down (2011) Rev Chil Pediatr. SciELO 82: 105-112.

6. Groisman B, Gili J, Giménez L, Poletta F, Bidondo MP, et al. (2017) Geographic clusters of congenital anomalies in Argentina. J Community Genet 8: 1-7.

7. Zergollern L, BegoviÄ D, FemeniÄ-Kes R (1983) Hemophilia A associated with Down's syndrome. Lijec Vjesn 105: 296-299. [Crossref]

8. Kaczorowska-Hac B, Wlazlowski M, Wierzba J, Balcerska A (2013) Haemophilia A associated with Down's syndrome. Ann Hematol 92: 841-842. [Crossref]

9. Rastogi P, Kumar N, Ahluwalia J, Das R, Panigrahi I (2017) The first case report of a patient with coexisting hemophilia B and Down syndrome. Blood Res 52: 75-76.

10. Webb D1, Roberts I, Vyas P (2007) Haematology of Down syndrome. Arch Dis Child Fetal Neonatal Ed 92: F503-507. [Crossref]

11. Petik B, Cevik MO, Sirik M, Colak D, Erturk SM (2016) Disappearing Inferior Vena Cava in A Pediatric Patient with Down Syndrome and Hereditary Thrombophilia. $J$ Belg Soc Radiol 22: 1-4.

12. Stöllberger C, Weiss S, Zlabinger G, Finsterer J (2012) Cryptogenic embolic stroke in a girl with a trisomy 21 mosaic. Acta Neurol Belg 112: 203-204. [Crossref]

13. Kurokami T, Takasawa R, Takeda S, Kurobe M, Takasawa K, et al. (2018) Venous thromboembolism in two adolescents with Down syndrome. Turk J Pediatr 60: 429432. [Crossref]

14. Baram TZ, Fishman MA (1985) 'Top of the basilar' artery stroke in an adolescent with Down's syndrome. Arch Neurol 42: 296. [Crossref]

15. Tarlaci S, Sagduyu A (2001) Cerebral venous thrombosis in Down's syndrome. Clin Neurol Neurosurg 103: 242-244.

16. Girolami A, Arias M, Sueldo E, Scoles G, Ferrari S, et al. (2016) First report of homozygous factor VII Padua (Arg304Gln) defect in a family from Argentina. Hematol Med Oncol 1: 1-5.

17. Girolami A, Sueldo E, Ferrari S, Guerrero G, Cosi E, et al. (2018) Report of the Second Patient with a Homozygous FVII Padua (Arg304GIn) Defect in Northern Argentina. M J Case 3: 036.

18. Manghat N, Hancock J, Walsh M, Puckett M, Noble R, et al. (2004) Thrombolysis for central venous occlusion causing bilateral chylothorax in a patient with down syndrome. J Vasc Interv Radiol 15: 511-515.

19. Williams M, Nand S (2003) Superior sagittal sinus thrombosis in a child with Down syndrome. J Paediatr Child Health 39: 226-228. 
Girolami A (2020) Arg304Gln (FVII Padua) coagulation disorder in a patient with Down Syndrome (trisomy 21): a remarkable observation from Argentina

20. Caner I, Olgun H, Buyukavci M, Tastekin A, Ors R (2006) A giant thrombus in the right ventricle of a newborn with Down syndrome: successful treatment with rt-PA. $J$ Pediatr Hematol Oncol 28: 120-122.

21. Lin Y, Ma L, Jiang WJ, Liu YM (2018) Progressive Venous Thrombosis in an 18-YearOld Man with Down Syndrome. Chin Med J (Engl) 131: 2480-2481. [Crossref]
22. Alioğlu B, Atac B, Baskın E, Özbek N (2007) Deep vein thrombosis in a child with Down syndrome: A case report. Turk J Haematol 24: 136-139. [Crossref]

23. Murdoch JC, Rodger JC, Rao SS, Fletcher CD, Dunnigan MG (1977) Down's syndrome: an atheroma-free model? $\mathrm{Br}$ Med $J$ 2: 226-228. [Crossref]

Copyright: (C2020 Girolami A. This is an open-access article distributed under the terms of the Creative Commons Attribution License, which permits unrestricted use, distribution, and reproduction in any medium, provided the original author and source are credited. 